\title{
Meta-Analysis of Invasive versus Non-Invasive Techniques to Predict Fluid Responsiveness by Passive Leg Raising in the Critically Ill
}

\author{
Xiang $\mathrm{Si}^{1}$, Daiyin Cao ${ }^{2}$, Jianfeng $\mathrm{Wu}^{1}$, Juan Chen ${ }^{1}$, Zimeng Liu ${ }^{1}$, Minying Chen ${ }^{1}$, \\ Ouyang Bin ${ }^{1}$, Xiangdong Guan ${ }^{1 *}$ \\ ${ }^{1}$ Department of Surgical Intensive Care Unit, The First Affiliated Hospital of Sun Yat-sen University, Guangzhou, China \\ ${ }^{2}$ Department of Critical Care Medicine, The Sixth Affiliated Hospital of Sun Yat-sen University, Guangzhou, China \\ Email: *guanxiangdong1962@163.com
}

How to cite this paper: Si, X., Cao, D.Y., Wu, J.F., Chen, J., Liu, Z.M., Chen, M.Y., Bin, O.Y. and Guan, X.D. (2016) Meta-Analysis of Invasive versus Non-Invasive Techniques to Predict Fluid Responsiveness by Passive Leg Raising in the Critically Ill. International Journal of Clinical Medicine, 7, 736-747

http://dx.doi.org/10.4236/ijcm.2016.711080

Received: October 11, 2016

Accepted: November 19, 2016

Published: November 22, 2016

Copyright $\odot 2016$ by authors and Scientific Research Publishing Inc. This work is licensed under the Creative Commons Attribution International License (CC BY 4.0).

http://creativecommons.org/licenses/by/4.0/

\section{(c) (i) Open Access}

\begin{abstract}
Objective: To analyze the accuracy and specificity of recent studies to compare the ability of predicting fluid responsiveness with Passive Leg Raising (PLR) by using invasive or non-invasive techniques during passive leg raising. Data Sources: MEDLINE, EMBASE and the Cochrane Database of Systematic Reviews were systematically searched. Study Selection: Clinical trials that reported the sensitivity, specificity and area under the receiver operating characteristic curve (AUC) between the responder and non-responder induced by passive leg raising and Volume Expansion (VE) in critical ill patients were selected. 246 studies were screened, 14 studies were included for data extraction, which met our inclusion criteria. Data Extraction: Data were abstracted on study characteristics, patient population, type and amount of VE, time of VE, definition of responders, position, techniques used for measuring hemodynamic change, number and percentage of responders, the correlation coefficient, sensitivity, specificity, best threshold and area under the ROC curve (AUC). Meta-analytic techniques were used to summarize the data. Data Synthesis: A total of 524 critical ill patients from 14 studies were analyzed. Data are reported as point estimate (95\% confidence intervals). The pooled sensitivity and specificity of invasive techniques were $80 \%(73 \%-85 \%)$ and $89 \%(84 \%-93 \%)$ respectively with the area under the sROC of 0.94 . While, the pooled sensitivity and specificity of non-invasive techniques were $88 \%(84 \%-92 \%)$ and $91 \%(86 \%-94 \%)$ respectively with the area under the sROC of 0.95 . The pooled DOR of invasive techniques was 32.2 (13.6 76.8), which was much lower than that of non-invasive techniques with the value of 64.3 (33.9 - 121.7). Conclusions: The hemodynamic indexes changes induced by PLR could reliably predict fluid responsiveness. Non-invasive hemodynamic techniques with their accuracy and safety can benefit the daily work in ICUs. Because the num-
\end{abstract}


ber of patients included in the present trials was small, further studies should be undertaken to confirm these findings.

\section{Keywords}

Invasive, Non-Invasive, Fluid Responsiveness, Meta-Analysis

\section{Introduction}

Fluid therapy is an essential part in Intensive Care Unit (ICU) to survive patients with hypovolemia. In fact, that's not easy. Studies have shown that about $50 \%$ of critically ill patients do not exhibit the desired effect [1]. How to assess intravascular volume accurately has been a critical problem.

Passive Leg Raising (PLR) is a reversible maneuver that mimics rapid Volume Expansion (VE) by shifting venous blood from the lower limbs toward the intrathoracic compartment [2]. Thus, PLR increases the cardiac preload. PLR has been validated to predict fluid responsiveness, but it requires the determination of $\mathrm{CO}$ or its surrogates with a fast-response device, because the hemodynamic changes may be transient [3] [4].

There are a lot of "fast-response devices" and all of them can be divided into 2 categories: invasive and non-invasive. Invasive hemodynamic techniques such as transpulmonarythermodilution (PiCCO), Vigileo, arterial BP transducer, pulmonary artery catheter are widely used in intensive units. Over the past few years, new techniques assessed for rapid and non-invasive prediction of fluid responsiveness have been introduced in clinical practice. Transthoracic echocardiography (TTE), transesophageal echocardiography (TEE), transthoracic Doppler ultrasonography (USCOM), Bioreactance technology-based system (NICOM), Continuous Non-invasive Arterial Pressure (CNAP) have been developed to predict fluid responsiveness.

Evidence shows that various studies have confirmed the ability of predicting fluid responsiveness by these techniques, but the predictive value of the hemodynamic response after PLR as a dynamic index of fluid responsiveness between invasive and noninvasive techniques has not been compared yet. The aim of this systematic review is to answer the question: can non-invasive techniques be better than invasive ones to be used as a tool for predicting volume responsiveness in critically ill during PLR maneuver and VE?

Data reporting conformed to the Standards for Reporting of Diagnostic Accuracy (STARD) [5].

\section{Materials and Methods}

\subsection{Search Strategy}

Two authors independently performed a search in MEDLINE (using PubMed as the search engine, from 1947), EMBASE (from 1974) and the Cochrane Database of Systematic Reviews for prospective studies in January 2014 with the following key words: 
"Passive leg raising" AND (fluid therapy OR fluid responsiveness OR fluid expansion OR fluid load ${ }^{\star}$ OR volume therapy OR volume responsiveness OR volume expansion).

\subsection{Study Selection}

Only full-text articles in indexed journals were included. Reviews, chapter, case reports, reference network and studies published in abstract form were excluded. No language restriction was imposed. We included only studies with patients admitted in intensive care unit (ICU). Children and pregnant women would be excluded. Articles were collected by one reviewer and crosschecked by another reviewer and references of included papers were examined to identify other studies of interest.

\subsection{Inclusion Criteria}

We included full-text studies with the following criteria: 1. PLR was performed and followed with VE; 2 . the number of patients and boluses had been counted; 3. the reference standard of predicting fluid responsiveness had been described; 4 . the number of responsive patients and non-responsive patients had been counted; 5 . sensitivity, specificity and the threshold of the index in identifying those patients who subsequently responded to VE (responders) had been calculated.

\subsection{Data Extraction and Quality Assessment}

Data were extracted using a structured data collection sheet including the following items: authors, year of publication, study setting, population, age of patients, number of patients included, ventilation mode, cardiac rhythm (sinus vs. arrhythmias), type and amount of VE, time of VE, definition of responders, position, assessments used for measuring hemodynamic change, number of VE administered, number and percentage of responders, sensitivity, specificity, best threshold and area under the ROC curve (AUC). We use QUADAS-2 (quality assessment of diagnostic accuracy-2) [6] to assess the quality of included studies on diagnostic accuracy in systematic reviews. The checklist was structured with 4 parts: patient selection, index test, reference standard and flow and timing.

\subsection{Statistical Analysis}

We used RevMan 5.2 (Cochrane Collaboration, Oxford, UK) to make the QUADAS-2 scale to assess quality of studies on diagnostic accuracy to be included in systematic reviews. To calculate pooled values of sensitivity, specificity, diagnostic odds ratio (DOR) and area under summary receiver operating characteristic (sROC) curve we used MetaDiSC 1.4 (Unit of Clinical Biostatisticsteam of the Ramon y Cajal Hospital, Madrid, Spain). P-values of less than 0.05 were considered statistically significant. Publication bias was performed by STATA statistical software 12.0 (StataCorp, College Station, TX).

We used the Cochran Q statistic [7] to evaluate heterogeneity between studies. When the value of $\mathrm{p}$ less than or equal to 0.10 and $\mathrm{I}^{2}$ more than $50 \%$, it could be regarded as 
heterogenerity significantly and a random effect model was used to perform metaanalysis. For sensitivity and specificity, the Spearman correlation coefficient between those two parameters was calculated to evaluate a threshold effect determining heterogeneity [8].

For each study, sensitivity, specificity, positive likelihood ratio (+LR), negative likelihood ratio ( $-\mathrm{LR})$, and DOR were calculated after constructing a $2 \times 2$ contingency table. Pooled estimates with $95 \%$ confidence intervals (CIs) were calculated using a random-effects model. A summary receiver operating characteristic (sROC) curve was drawn according to the regression model proposed by Moses et al. [9] and it was performed to assess the interaction between sensitivity and specificity. The area under the sROC curve (AUC) was obtained to assess the diagnostic performance of hemodynamic techniques. Potential presence of publication bias was tested using the Egger [10] and Begg test [11].

\section{Results}

\subsection{Process of Study Selection}

The initial search yielded 246 articles after the first query in the three databases. Among them, 86 were excluded for not directly concerning item of interest. In the 160 full-articles, 103 were excluded because they were reviews, chapters or abstracts. 16 were excluded because they didn't perform PLR and another 14 were excluded because they didn't use VE. 13 were excluded because they didn't satisfy our inclusion criteria. Therefore, 14 studies [12]-[25] were included for final analysis.

\subsection{Characteristics of Included Studies}

The clinical characteristics of the 14 included studies were summarized in Table 1 and main results were reported in Table 2 and Table 3. The results of QUADAS-2 were showed in Figure 1. All the included 14 studies were prospective studies with enrollment of patients with sign of inadequate tissue perfusion. We found good compliance with appropriate population selection, index test adequately described, appropriate reference standard, and adequate flow and timing. Population selection bias was minimized, as the inclusion criteria of the patients were close. However, no study described the blinding of the assessors to the outcome measurement of the results. 2 out of the 14 included studies didn't report the lasting time of PLR.

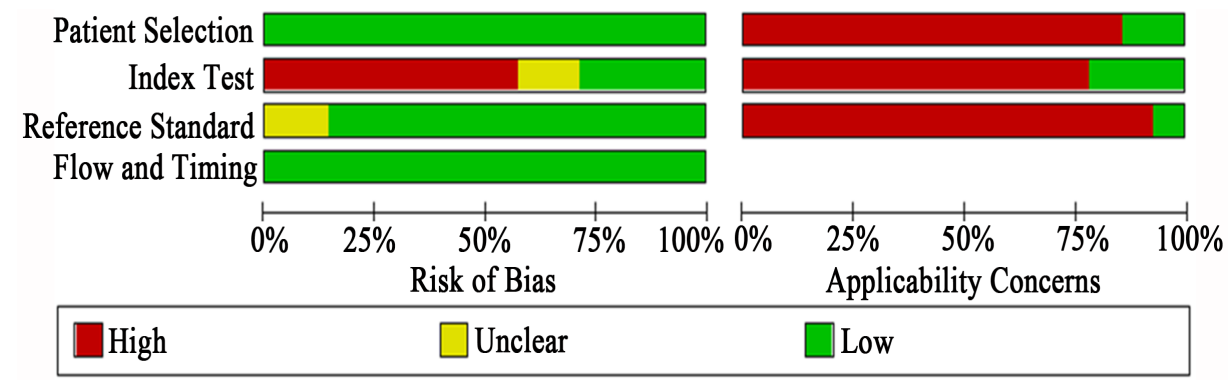

Figure 1. Results of QUADAS-2 (software RevMan 5.2). 
Table 1. Main characteristics of the included studies.

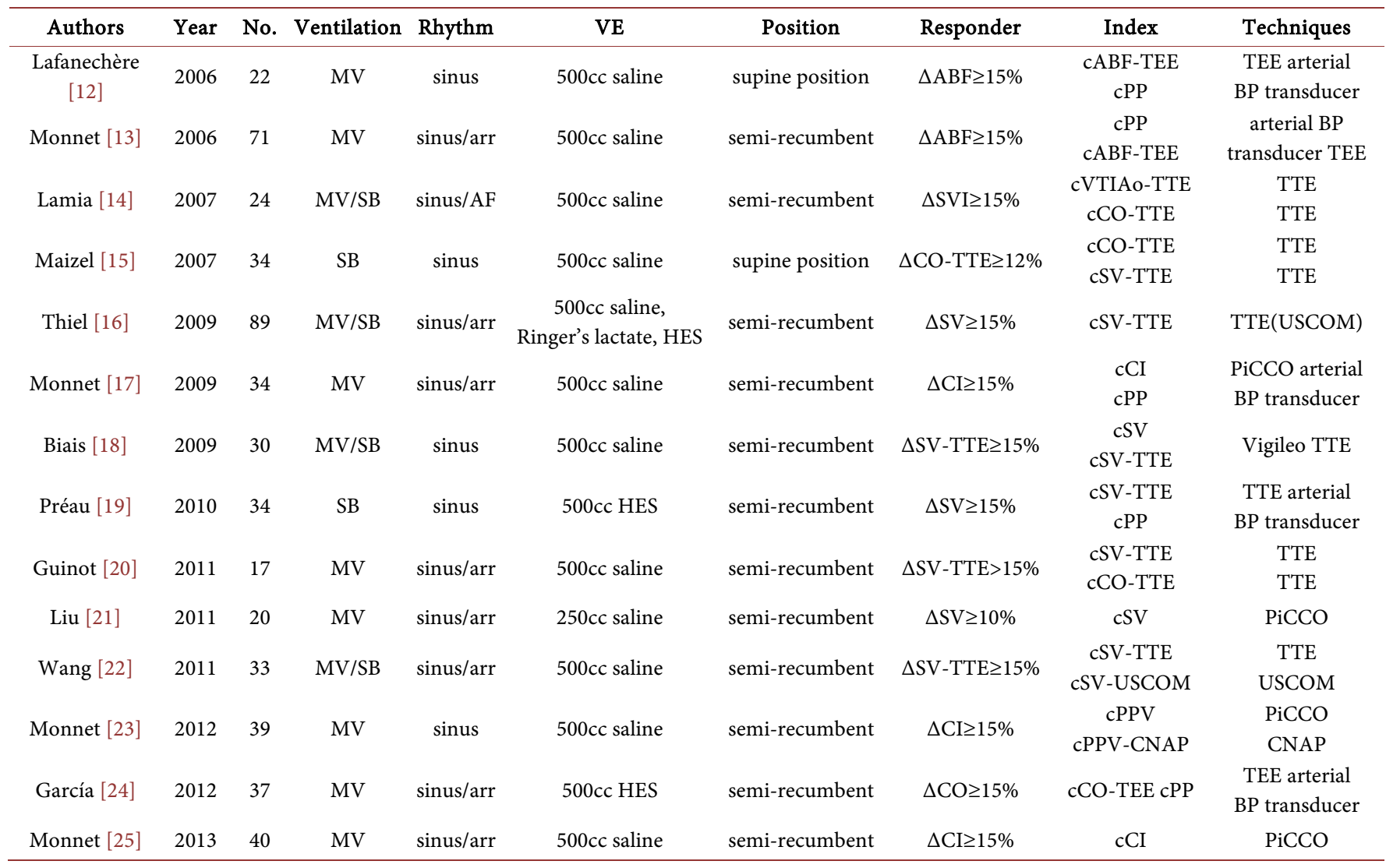

MV: mechanical ventilation, arr: arrhythmia, AF: atrial fibrillation, VE: volume expansion, min minutes, BP: blood pressure, $\Delta$ : variation; c: PLR-induced changes, TTE: transthoracic echocardiography, TEE: transesophageal echocardiography, CI: cardiac index, CO: cardiac output, SV: stroke volume, PP: pulse pressure, PPV: pulse pressure variation, ABF: aortic blood flow, VTIAo: aortic velocity-time integral, USCOM: transthoracic Doppler ultrasonography, CNAP: continuous noninvasive arterial pressure.

Table 2. Pooled results for predictive capacity of invasive hemodynamic techniques.

\begin{tabular}{|c|c|c|c|c|c|c|c|c|c|c|c|c|c|}
\hline Authors & Index & Boluses & TP & FP & FN & TN & AUC & Best Threshold & Sens. & Spec. & DOR & $+\mathrm{LR}$ & $-\mathrm{LR}$ \\
\hline Lafanechère [12] & cPP & 22 & 7 & 1 & 3 & 11 & 0.78 & 12 & 70 & 92 & 25.7 & 8.4 & 0.3 \\
\hline Monnet [13] & $\mathrm{cPP}$ & 71 & 22 & 5 & 15 & 29 & 0.96 & 12 & 60 & 85 & 8.5 & 4 & 0.5 \\
\hline Monnet [17] & $\mathrm{cCI}$ & 34 & 21 & 0 & 2 & 11 & 0.94 & 10 & 91 & 100 & 197.8 & 21.5 & 0.1 \\
\hline Biais [18] & $\mathrm{cSV}$ & 30 & 20 & 2 & 0 & 8 & 0.96 & 13 & 100 & 80 & 139.4 & 4.3 & 0 \\
\hline Monnet [23] & $\mathrm{cPPV}$ & 39 & 15 & 2 & 2 & 20 & 0.89 & 10 & 88 & 91 & 75 & 9.7 & 0.1 \\
\hline García [24] & $\mathrm{cPP}$ & 37 & 14 & 3 & 7 & 13 & 0.73 & 11 & 67 & 81 & 8.7 & 3.6 & 0.4 \\
\hline Monnet [25] & cCI & 40 & 20 & 1 & 1 & 18 & 0.98 & 15 & 95 & 95 & 360 & 18.1 & 0.1 \\
\hline Overall (95\% CIs) & & 353 & & & & & & & 80 & 89 & 32.2 & 5.8 & 0.2 \\
\hline
\end{tabular}

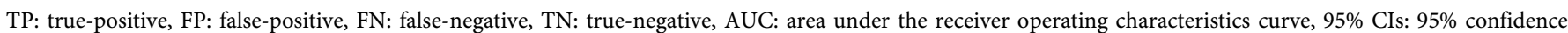

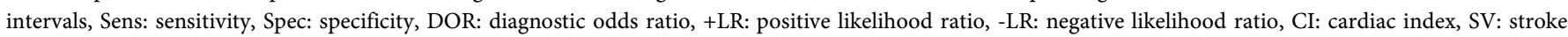
volume, PP: pulse pressure, PPV: pulse pressure variation. 
Table 3. Pooled results for predictive capacity of non-invasive hemodynamic techniques.

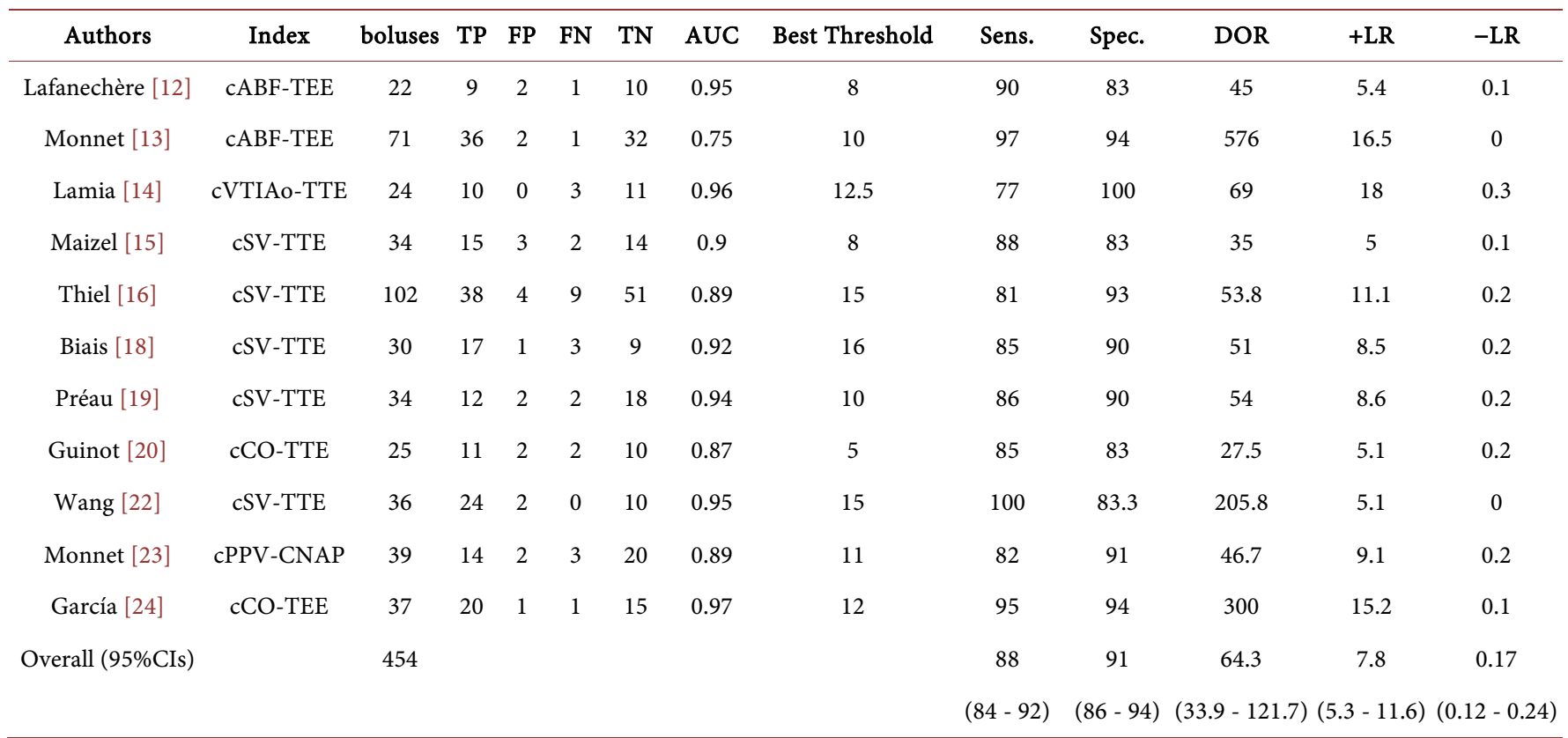

TP: true-positive, FP: false-positive, FN: false-negative, TN: true-negative, AUC: area under the receiver operating characteristics curve, $95 \%$ CIs: $95 \%$ confidence intervals, Sens: sensitivity, Spec: specificity, DOR: diagnostic odds ratio, SV: stroke volume, ABF: aortic blood flow, VTIAo: aortic velocity-time integral, TTE: transthoracic echocardiography, TEE: transesophageal echocardiography, CNAP: continuous non-invasive arterial pressure.

A total of 524 patients were enrolled (range $17-89$ for single paper) and a total of $574 \mathrm{VE}$ were administered. The mean responder rate was $52.8 \%$.

All studies were conducted in intensive care units (ICU) on patients with hypovolemia, whose attending physician decided to perform a fluid challenge. 2 study [15] [19] enrolled patients had spontaneous breathing without mechanical ventilator in sinus rhythm. The others [12] [13] [14] [16] [17] [18] [20]-[25] enrolled patients with mechanical ventilation and/or arrhythmias. The reference standard for definition of responders after fluid bolus as $\mathrm{CO}$ or its surrogates ranged between 10 and $15 \%$. 11 out of 14 studies [12] [13] [14] [15] [17] [18] [20] [21] [22] [23] [25] used saline for volume expansion (VE). 2 studies [19] [24] used hetastarch for VE. Only 1 study used either saline, ringer's lactate or hetastarch for VE. PLR was starting from a supine position in 2 studies [12] [15], and from semi-recumbent position in 12 studies [13] [14] [16]-[25]. 9 studies [12] [13] [17] [18] [19] [21] [23] [24] [25] used invasive hemodynamic techniques like PiCCO, Vigileo and arterial BP transducer and 11 studies [12] [13] [14] [15] [16] [18] [19] [20] [22] [23] [24] used non-invasive techniques, such as TEE, TTE, NICOM, USCOM and CNAP.

\subsection{Diagnostic Accuracy of Invasive Techniques}

We first divided the 14 studies into 2 groups: invasive group [12] [13] [17] [18] [19] [21] [23] [24] [25] and non-invasive group [12] [13] [14] [15] [16] [18] [19] [20] [22] [23] [24]. Then we meta-analyzed all papers into each group. Results were reported in Table 2 and Table 3 . When a study used both invasive and non-invasive techniques 
[12] [13] [18] [19] [23] [24], the indices of both techniques could be included. When a study reported analysis for two indices of the same category [14] [15] [17] [20] [21] [22] reported by the same technique only one was included in the meta-analysis in order to avoid duplication of sample size.

There were 9 papers ( 327 patients, 353 boluses) in the invasive group. The results $\mathrm{I}^{2}=$ $39.6 \%(<50 \%)$ and $p=0.1037(>0.05)$ showed that heterogeneity was not significant among the trials. Forest plots of the pooled sensitivity and specificity were shown in Figure 2 . The sensitivity ranged from $60 \%-100 \%$ (pooled sensitivity $80 \%, 95 \%$ CI: $73 \%$ - 85\%), while specificity ranged from $85 \%$ - 100\% (pooled specificity $89 \%, 95 \%$ CI: $84 \%$ - 93\%). DOR was 32.2 (95\% CI: 13.5 - 76.8). Pooled values for positive likelihood ratio (+LR) and negative likelihood ratio (-LR) were 5.8 (95\% CI: 3.8 - 8.8) and 0.2 (95\% CI: $0.1-0.4)$. The threshold for predicting fluid responsiveness varied between $9 \%$ and $15 \%$.

After excluded the threshold effect with spearman correlation coefficient $=0.233$ and $p=0.546$ (>0.05), we used Moses-Shapiro-Littenberg method to draw the symmetrical summary ROC curve (SROC) with AUC of 0.94 .

\subsection{Diagnostic Accuracy of Non-Invasive Techniques}

There were 11 papers (430 patients, 454 boluses) in the non-invasive group. The results

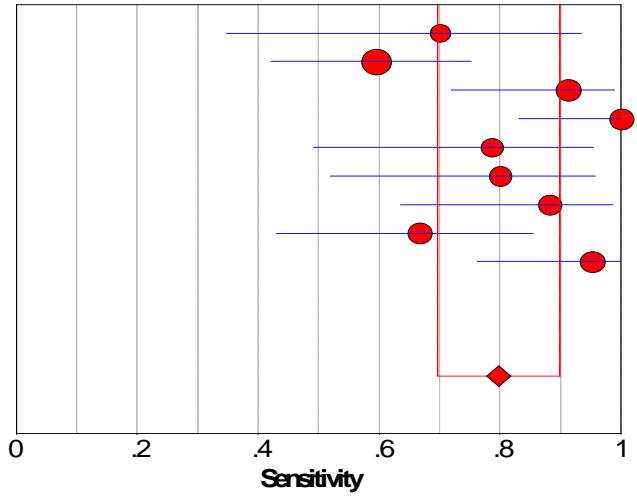

Lafanedhère(11)

Momet (12)

Momet (16)

Biais (17)

Préau(18)

Lu(20)

Mornet (22)

García(23)

Mornet (24)

Poded Sensitivity $=0.80(0.70$ to 0.90$)$

Chi-square $=26.83$, of $=8(p=0.0008)$

Inoonsistency $(1-$ square $)=70.2 \%$

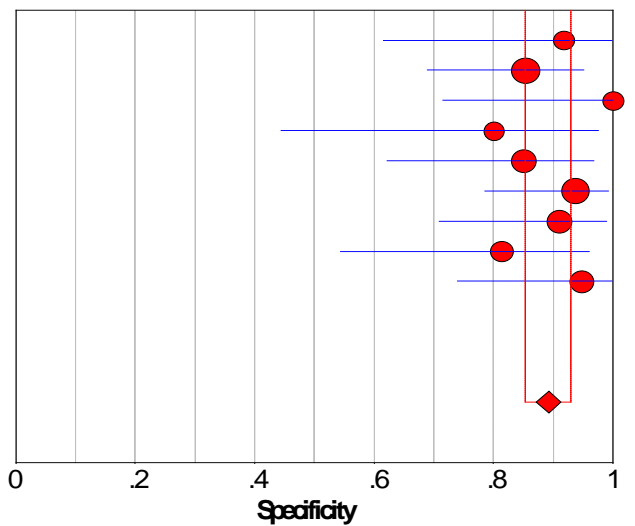

Lafanedhère(11)

Momet (12)

Mornet (16)

Bais(17)

Préau(18)

Liu(20)

Monnet (22)

Gróa(23)

Munet (24)

Specificity (95\%)

$0.92(0.62-10)$

$0.85(0.69-0.95)$

$10(0.72-10)$

$0.80(0.44-0.97)$

$0.85(0.62-0.97)$

$0.94(0.79-0.99)$

$0.91 \quad(0.71-0.99)$

$0.81(0.54-0.96)$

$0.95(0.74-10)$

Poded Speaifiaty $=0.89(0.85$ to 0.93$)$

Ci-square $=6.52 ; \mathrm{df}=8(\mathrm{p}=0.5899)$

Inconsistenoy (-square) $=0.0 \%$

Figure 2. Forest plots of pooled sensitivity between invasive and non-invasive techniques. 
$\mathrm{I}^{2}=0.0 \%(<50 \%)$ and $p=0.809(>0.05)$ showed that heterogeneity was not significant. Forest plots of the pooled sensitivity and specificity were shown in Figure 3 . The sensitivity ranged from $77 \%-100 \%$ (pooled sensitivity $88 \%$, 95\% CI: $84 \%-92 \%$ ), while specificity ranged from $83 \%-100 \%$ (pooled specificity $91 \%$, 95\% CI: $86 \%-94 \%$ ). DOR was 64.3 (95\% CI: 33.9 - 121.7). Pooled values for positive likelihood ratio (+LR) and negative likelihood ratio (-LR) were 7.8 (95\% CI: 5.3 - 11.6) and 0.2 (95\% CI: 0.1 - 0.2). The threshold for predicting fluid responsiveness varied between 5 and 15\%.

After excluded the threshold effect with spearman correlation coefficient $=0.361$ and $p=0.276$ (>0.05), we drew the symmetrical summary ROC curve (SROC) (Figure 4), with AUC of 0.95 .

\subsection{Publication Bias}

The result of Egger test and Begg test showed that the potential publication bias was significant $(P>0.05)$, which indicated a potential for publication bias.

\section{Discussion}

The main finding of our systematic review are as follows: (1) The result of pooled sensi-

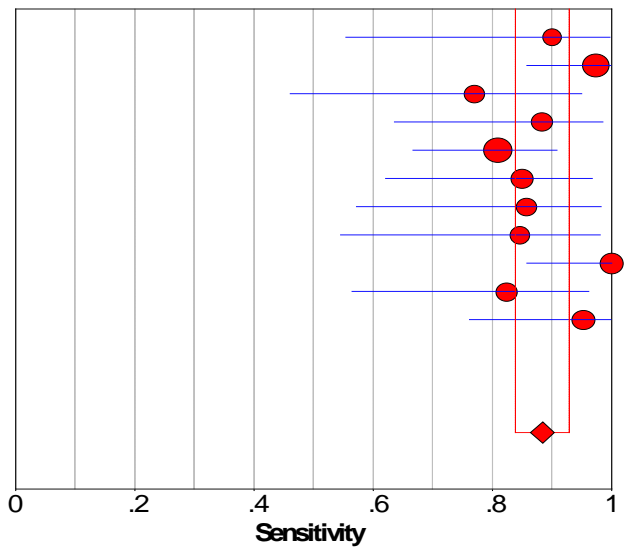

Lafanechère (11)

Monnet (12)

Lamia (13)

Maizel (14)

Thiel (15)

Biais (17)

Préau (18)

Ginot (19)

Wang (21)

Monnet (22)

Sensitivity $(95 \% \mathrm{C})$

$0.90(0.55-1.00)$

$0.97(0.86-1.00)$

$0.77 \quad(0.46-0.95)$

$0.88(0.64-0.99)$

$0.81 \quad(0.67-0.91)$

$0.85(0.62-0.97)$

$0.86(0.57-0.98)$

$0.85(0.55-0.98)$

$1.00(0.86-1.00)$

$0.82(0.57-0.96)$

$0.95(0.76-1.00)$

Pooled Sensitivity $=0.88$ (0.84 to 0.93 )

Chi-square $=15.72 ;$ df $=10(p=0.1079)$

Inconsistency (I-square) $=36.4 \%$

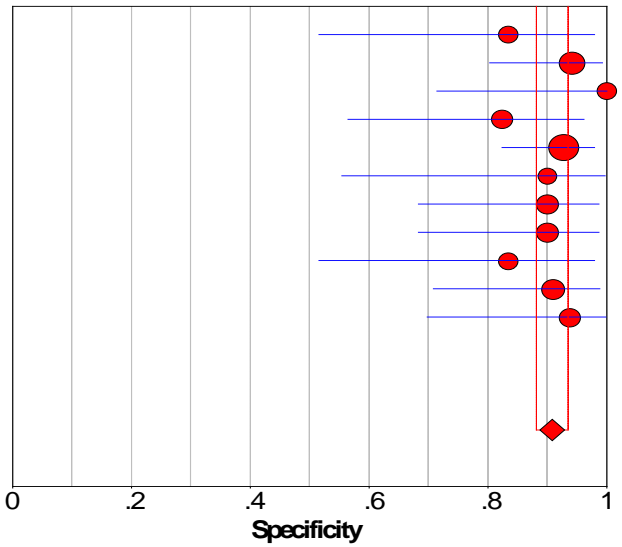

Lafanechère (11)

Monnet (12)

Lamia(13)

Maizel (14)

Thiel (15)

Biais (17)

Préau (18)

Guinot (19)

Wang (21)

Monnet (22)

García (23)

Specificity (95\%C)

$0.83(0.52-0.98)$

$0.94(0.80-0.99)$

$1.00(0.72-100)$

$0.82(0.57-0.96)$

$0.93(0.82-0.98)$

$0.90(0.55-100)$

$0.90(0.68-0.99)$

$0.90(0.68-0.99)$

$0.83 \quad(0.52-0.98)$

$0.91 \quad(0.71-0.99)$

$0.94 \quad(0.70-100)$

Pooled Specificity $=0.91$ (0.88 to 0.93 )

Chi-square $=5.61 ;$ df $=10(p=0.8468)$

Inconsistency (I-square) $=0.0 \%$

Figure 3. Forest plots of pooled specificity between invasive and non-invasive techniques. 


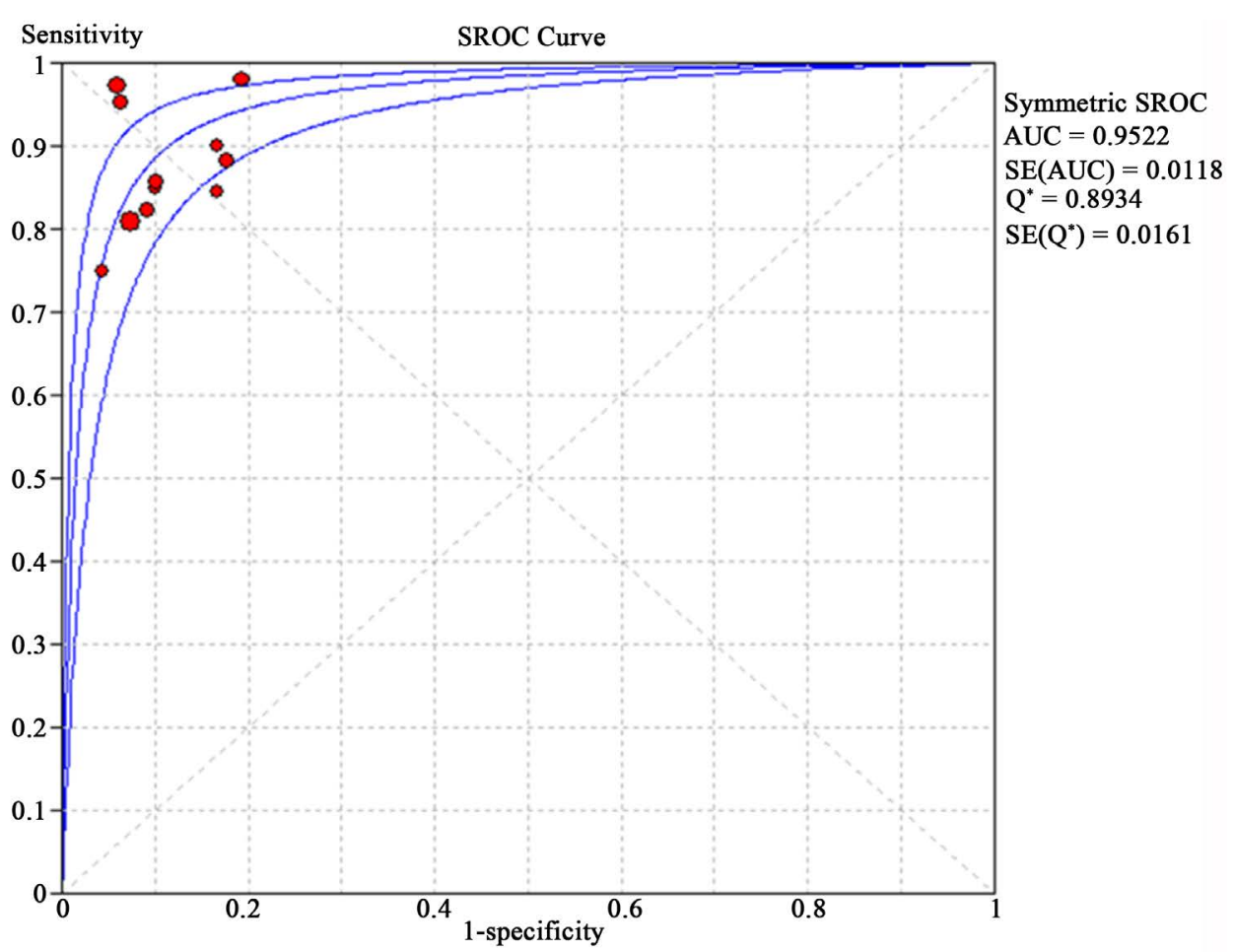

Figure 4. Summary receiver operating characteristics curve for the ability of non-invasive techniques discriminate between responders and non-responders.

tivity and specificity between invasive and non-invasive techniques are $80 \%$ (73\% $85 \%)$ vs. $88 \%(84 \%-92 \%)$ and $89 \%(84 \%-93 \%)$ vs. $91 \%(86 \%-94 \%)$, which cannot conclude inferior or superior; (2) The results of pooled DOR between invasive and non-invasive is 32.2 (13.6 - 76.8) vs. 64.3 (33.9 - 121.7), which indicate using non-invasive techniques have better discriminatory test performance with higher DOR values [8].

Knowing that dynamic indexes such as CO, CI, SV, ABF, SVV, PPV make use of provoked cardiac reaction assessed with fluid bolus and postural change can predict fluid responsiveness. A recent analysis by Vallee $\mathrm{F}$ shows that increase in thermodilution $\mathrm{CO}$ following a fluid bolus can predict fluid responsiveness [26]. The invasive techniques such as PiCCO, Vigileo, and arterial BP transducer are widely used in ICU to assess the patients' volume statue. Also, a systematic review by Mandeville et al. [27] assessed the value of TTE in predicting fluid responsiveness in critically ill. In our review, both invasive and non-invasive hemodynamic techniques can accurately predict fluid responsiveness. DOR is the ratio of the odds of positive test results between the diseased and nondiseased groups. Non-invasive techniques have higher values of DOR can better discriminate test performance. Importantly, non-invasive techniques are much safer, more convenient than invasive ones. But the non-invasive techniques, especially for TTE and TEE require an experienced echocardiography practitioner, who can take echocardiography pictures to answer clinical questions arising in critical illness. Jensen showed that with only limited training, a diagnostic transthoracic window 
was achieved 97 percent of the time when used in the evaluation of shock [28].

\section{Strengths and Limitations}

The strengths of our meta-analysis lie in the methods adhering to recent guidelines for diagnostic reviews [6] [29] [30], as well as in the advanced statistical methods used [31], which analyze all reported thresholds, sensitivity, specificity and their correlated results simultaneously. Also no other review has compared the ability to predict fluid responsiveness between these 2 groups of hemodynamic techniques. The results of our review could guide the using of the techniques to assess patients' volume statue in our clinical practice.

Limitations still exist in our meta-analysis. First, the pooling of diagnostic accuracy data inevitably contributed to sources of bias [7], which could be revealed in the significant amount of statistical heterogeneity across studies. Second, the number of patients included in the present trials was small (14 studies, 524 patients). A better review needs larger sample of studies. Third, the criteria of the included studies are based on clinical manifestation and the confounding factors such as cardiac function, respiratory function, severity of disease have not been analyzed.

\section{Conclusion}

The hemodynamic indexes induced by PLR can well discriminate between fluid responders and non-responders regardless of arrhythmia and ventilation mode. Non-invasive hemodynamic techniques with their accuracy and safety can benefit the daily work in ICUs.

\section{References}

[1] Michard, F. and Teboul, J.L. (2002) Predicting fluid responsiveness in ICU patients: a critical analysis of the evidence. Chest, 121, 2000-2008.

http://dx.doi.org/10.1378/chest.121.6.2000

[2] Rutlen, D.L., Wackers, F.J. and Zaret, B.L. (1981) Radionuclide assessment of peripheral intravascular capacity: A technique to measure intravascular volume changes in the capacitance circulation in man. Circulation, 64, 146-152.

http://dx.doi.org/10.1161/01.CIR.64.1.146

[3] Marik, P.E., Monnet, X. and Teboul, J.L. (2011) Hemodynamic Parameters to Guide Fluid Therapy. Annals of Intensive Care, 1, 1. http://dx.doi.org/10.1186/2110-5820-1-1

[4] Monnet, X. and Teboul, J.L. (2008) Passive Leg Raising. Intensive Care Medicine, 34, 659663. http://dx.doi.org/10.1007/s00134-008-0994-y

[5] Bossuyt, P.M., Reitsma, J.B., Bruns, D.E., et al. (2003) The STARD Statement for Reporting Studies of Diagnostic Accuracy: Explanation and Elaboration. Annals of Internal Medicine, 138, W1-W12. http://dx.doi.org/10.7326/0003-4819-138-1-200301070-00012-w1

[6] Whiting, P.F., Rutjes, A.W.S., Westwood, M.E., et al. (2011) QUADAS-2: A Revised Tool for the Quality Assessment of Diagnostic Accuracy Studies. Annals of Internal Medicine, 155, 529-536. http://dx.doi.org/10.7326/0003-4819-155-8-201110180-00009

[7] Whiting, P., Rutjes, A.W., Reitsma, J.B., Glas, A.S., Bossuyt, P.M.M. and Kleijnen, J. (2004) Sources of Variation and Bias in Studies of Diagnostic Accuracy: A Systematic Review. An- 
nals of Internal Medicine, 140, 189- 202.

http://dx.doi.org/10.7326/0003-4819-140-3-200402030-00010

[8] Devillé, W.L., Buntinx, F., Bouter, L.M., et al. (2002) Conducting Systematic Reviews of Diagnostic Studies: Didactic Guidelines. BMC Medical Research Methodology, 2, 9.

http://dx.doi.org/10.1186/1471-2288-2-9

[9] Moses, L.E., Shapiro, D. and Littenberg, B. (1993) Combining independent Studies of a Diagnostic Test into a Summary ROC Curve: Data-Analytic Approaches and Some Additional Considerations. Statistics in Medicine, 12, 1293-1316. http://dx.doi.org/10.1002/sim.4780121403

[10] Egger, M., Smith, G.D., Schneider, M. and Minder, C. (1997) Bias in Meta-Analysis Detected by a Simple, Graphical Test. BMJ, 315, 629-634.

http://dx.doi.org/10.1136/bmj.315.7109.629

[11] Begg, C.B. and Mazumdar, M. (1994) Operating Characteristics of a Rank Correlation Test for Publication Bias. Biometrics, 1088-1101. http://dx.doi.org/10.2307/2533446

[12] Lafanechere, A., Pene, F., Goulenok, C., et al. (2006) Changes in Aortic Blood Flow Induced by Passive Leg Raising Predict Fluid Responsiveness in Critically Ill Patients. Critical Care, 10, R132. http://dx.doi.org/10.1186/cc5044

[13] Monnet, X., Rienzo, M., Osman, D., Anguel, N., Richard, C., Pinsky, M.R. and Teboul, J.L. (2006) Passive Leg Raising Predicts Fluid Responsiveness in the Critically Ill. Critical Care Medicine, 34, 1402-1407. http://dx.doi.org/10.1097/01.CCM.0000215453.11735.06

[14] Lamia, B., Ochagavia, A., Monnet, X., et al. (2007) Echocardiographic Prediction of Volume Responsiveness in Critically Ill Patients with Spontaneously Breathing Activity. Intensive Care Medicine, 33, 1125-1132. http://dx.doi.org/10.1007/s00134-007-0646-7

[15] Maizel, J., Airapetian, N., Lorne, E., et al. (2007) Diagnosis of Central Hypovolemia by Using Passive Leg Raising. Intensive Care Medicine, 33, 1133-1138. http://dx.doi.org/10.1007/s00134-007-0642-y

[16] Thiel, S., Kollef, M. and Isakow, W. (2009) Non-Invasive Stroke Volume Measurement and Passive Leg Raising Predict Volume Responsiveness in Medical ICU Patients: An Observational Cohort Study. Critical Care, 13, R111. http://dx.doi.org/10.1186/cc7955

[17] Monnet, X., Osman, D., Ridel, C., et al. (2009) Predicting Volume Responsiveness by Using the End-Expiratory Occlusion in Mechanically Ventilated Intensive Care Unit Patients. Critical Care Medicine, 37, 951-956. http://dx.doi.org/10.1097/CCM.0b013e3181968fe1

[18] Biais, M., Vidil, L., Sarrabay, P., et al. (2009) Changes in Stroke Volume Induced by Passive Leg Raising in Spontaneously Breathing Patients: Comparison between Echocardiography and Vigileo/FloTrac Device. Critical Care, 13, R195. http://dx.doi.org/10.1186/cc8195

[19] Préau, S., Saulnier, F., Dewavrin, F., et al. (2010) Passive Leg Raising Is Predictive of Fluid Responsiveness in Spontaneously Breathing Patients with Severe Sepsis or Acute Pancreatitis. Critical Care Medicine, 38, 819-825. http://dx.doi.org/10.1097/CCM.0b013e3181c8fe7a

[20] Guinot, P.G., Zogheib, E., Detave, M., et al. (2011) Passive Leg Raising Can Predict Fluid Responsiveness in Patients Placed on Venovenous Extracorporeal Membrane Oxygenation. Critical Care, 15, R216. http://dx.doi.org/10.1186/cc10451

[21] Liu, Y., Lu, Y.H., Xie, J.F., Qiu, X.H., et al. (2011) Passive Leg Raising Predicts Volume Responsiveness in Patients with Septic Shock. Chinese Journal of Surgery, 49, 44-48.

[22] Wang, H.L., Liu, H.T. and Yu, K.L. (2011) Clinical Observation of Passive Leg Raising Combined with Non Invasive Cardiac Output Monitoring System in Predicting Volume Responsiveness. Chinese Critical Care Medicine, 23, 146-149. 
[23] Monnet, X., Dres, M., Ferre, A., et al. (2012) Prediction of Fluid Responsiveness by a Continuous Non-Invasive Assessment of Arterial Pressure in Critically Ill Patients: Comparison with Four Other Dynamic Indices. British Journal of Anaesthesia, 109, 330-338. http://dx.doi.org/10.1093/bja/aes182

[24] García, M.I.M., Cano, A.G., Romero, M.G., et al. (2012) Non-Invasive Assessment of Fluid Responsiveness by Changes in Partial End-Tidal $\mathrm{CO}_{2}$ Pressure during a Passive Leg-Raising Maneuver. Annals of Intensive Care, 2, 9. http://dx.doi.org/10.1186/2110-5820-2-9

[25] Monnet, X., Bataille, A., Magalhaes, E., et al. (2013) End-Tidal Carbon Dioxide Is Better than Arterial Pressure for Predicting Volume Responsiveness by the Passive Leg Raising Test. Intensive Care Medicine, 39, 93-100. http://dx.doi.org/10.1007/s00134-012-2693-y

[26] Vallee, F., Mari, A., Perner, A. and Vallet, B. (2010) Combined Analysis of Cardiac Output and CVP Changes Remains the Best Way to Titrate Fluid Administration in Shocked Patients. Intensive Care Medicine, 36, 912-914. http://dx.doi.org/10.1007/s00134-010-1831-7

[27] Mandeville, J.C. and Colebourn, C.L. (2012) Can Transthoracic Echocardiography Be Used to Predict Fluid Responsiveness in the Critically Ill Patient? A Systematic Review. Critical Care Research and Practice, 2012, Article ID: 513480. http://dx.doi.org/10.1155/2012/513480

[28] Jensen, M.B., Sloth, E., Larsen, K.M., et al. (2004) Transthoracic Echocardiography for Cardiopulmonary Monitoring in Intensive Care. European journal of Anaesthesiology, 21, 700 707. http://dx.doi.org/10.1097/00003643-200409000-00006

[29] Irwig, L., Tosteson, A.N., Gatsonis, C., Lau, J., Colditz, G., Chalmers, T.C., et al. (1994) Guidelines for Meta-Analyses Evaluating Diagnostic Tests. Annals of Internal Medicine, 120, 667-676. http://dx.doi.org/10.7326/0003-4819-120-8-199404150-00008

[30] Khan, K.S., Dinnes, J. and Kleijnen, J. (2001) Systematic Reviews to Evaluate Diagnostic Tests. European Journal of Obstetrics \& Gynecology and Reproductive Biology, 95, 6-11. http://dx.doi.org/10.1016/S0301-2115(00)00463-2

[31] Riley, R.D. (2009) Multivariate Meta-Analysis: The Effect of Ignoring Within-Study Correlation. Journal of the Royal Statistical Society: Series A, 172, 789-811. http://dx.doi.org/10.1111/j.1467-985X.2008.00593.x

Submit or recommend next manuscript to SCIRP and we will provide best service for you:

Accepting pre-submission inquiries through Email, Facebook, LinkedIn, Twitter, etc.

A wide selection of journals (inclusive of 9 subjects, more than 200 journals)

Providing 24-hour high-quality service

User-friendly online submission system

Fair and swift peer-review system

Efficient typesetting and proofreading procedure

Display of the result of downloads and visits, as well as the number of cited articles

Maximum dissemination of your research work

Submit your manuscript at: http://papersubmission.scirp.org/

Or contact ijcm@scirp.org 